\title{
S-allylcysteine induces cell cycle arrest and apoptosis in androgen-independent human prostate cancer cells
}

\author{
ZHUO LIU, MINGCHAO LI, KE CHEN, JUN YANG, RUIBAO CHEN, \\ TAO WANG, JIHONG LIU, WEIMIN YANG and ZHANGQUN YE \\ Department of Urology, Tongji Hospital, Tongji Medical College, Huazhong University \\ of Science and Technology, Wuhan, Hubei 430030, P.R. China
}

Received August 11,2011; Accepted October 26, 2011

DOI: $10.3892 / \mathrm{mmr} .2011 .658$

\begin{abstract}
To increase the use of phytochemical supplements as chemoprevention or adjuvant drugs in cancer treatment, it is necessary to verify their biological effects and correlative mechanisms. Recently, S-allylcysteine (SAC) was identified as a potent compound derived from garlic. The aim of this study was to evaluate the anticancer effects of SAC on androgenindependent human prostate cancer (PC-3) cells and to elucidate the possible mechanisms. PC-3 cells were incubated with SAC at three different concentrations. Cell growth was determined by Cell Counting Kit-8 and 5-ethynyl-2'-deoxyuridine assay. Cell cycle and apoptosis were determined by flow cytometric assay. The expression of apoptosis-related molecules was detected by Western blot analysis. We found that SAC suppressed the proliferation of PC-3 cells and led to cell cycle arrest at the G0/G1 phases, as well as inducing cell apoptosis which was accompanied by the decreased expression of Bcl-2 and increased expression of Bax and caspase 8 . This study demonstrated the chemopreventive activity of SAC in vitro, and that SAC may be a promising candidate for prostate cancer treatment.
\end{abstract}

\section{Introduction}

Prostate cancer is the most commonly diagnosed malignancy and continues to be one of the leading causes of cancer-related mortality in American men (1). The incidence of prostate cancer in China is also rising year by year (2). Prostate cancers usually progress from androgen-dependent to androgenindependent with highly metastatic properties (3). The main treatments for advanced prostate cancers are hormonal treatments, chemotherapy or radiation. Unfortunately, these

Correspondence to: Dr Tao Wang, Department of Urology, Tongji Hospital, Tongji Medical College, Huazhong University of Science and Technology, Wuhan, Hubei 430030, P.R. China

E-mail: twang@tjh.tjmu.edu.cn

Key words: prostate cancer, PC-3 cells, S-allylcysteine, proliferation, apoptosis treatments do not appear to offer sufficient benefit in terms of improving the patient survival rate $(4,5)$. Dietary phytochemicals are a promising group of chemopreventive agents due to their low toxicity and their health benefits associated with other chronic diseases (6). Therefore, phytochemicals have been recognized as a new preventative and therapeutic approach for prostate cancer in recent years (7).

Allium vegetables, such as garlic, have been used for medicinal purposes throughout recorded history (8). Recent scientific investigations have shown that garlic has antioxidative (9), antihepatotoxic (10) and antihypertensive components (11), and also inhibits carcinogenesis at a variety of sites (12-14). S-allylcysteine (SAC) is the most abundant organosulfur compound in aged garlic extract, and is reported to have multiple functions, including anticancer effects (15-18). In the prostate cancer context, Chu et al reported that SAC demonstrated antiproliferation effects in prostate cell lines and pro-apoptotic effects in a CWR22R prostate cancer xenograft $(17,18)$. However, the mechanisms of the anticancer effects of SAC are not clear.

In the present study, to further determine the role of SAC as an anticancer phytochemical and explore the possible molecular mechanisms of this, the PC-3 cell line, a cell model for androgen-independent prostate cancer, was treated with various concentrations of SAC. Cell growth characteristics, including proliferation, cell cycle duration, apoptosis and the expression of apoptosis-related molecules, were evaluated. We found that SAC suppressed the proliferation of PC-3 cells and led to cell cycle arrest at the G0/G1 phases, as well as inducing cell apoptosis which was accompanied with the decreased expression of Bcl-2 and increased expression of Bax and caspase 8. Our results demonstrated for the first time that SAC induces cell cycle arrest in prostate cells.

\section{Materials and methods}

Reagents, antibodies and SAC preparation. RPMI-1640 medium and supplements were obtained from Invitrogen (Grand Island, NY, USA). Fetal bovine serum (FBS) was obtained from Hyclone (Logan, UT, USA). Cell Counting Kit-8 (CCK-8) was purchased from Dojindo (Kumamoto, Japan). 5-Ethynyl-2'-deoxyuridine (EdU) assay kit was obtained from Ribobio Co., Ltd. (Guangzhou, China). Antibodies to 
Bcl-2, Bax and caspase 8 were purchased from Santa Cruz Biotechnology (Santa Cruz, CA, USA). SAC was purchased from TCI Chemical Co. (Tokyo, Japan). A stock solution of SAC $(100 \mathrm{mM})$ was prepared freshly in distilled water, and added to the culture medium at a calculated final concentration of 5,10 and $15 \mathrm{mM}$.

Cell culture. The human androgen-independent prostate cancer cell line PC-3 was obtained from the American Type Culture Collection (ATCC; Manassas, VA, USA). PC-3 cells were grown in RPMI-1640 medium containing 5\% (v/v) FBS, $0.37 \%(\mathrm{w} / \mathrm{v}) \mathrm{NaHCO}_{3}$, penicillin $(100 \mathrm{U} / \mathrm{ml})$ and streptomycin $(100 \mathrm{U} / \mathrm{ml})$ in a humidified incubator with $5 \% \mathrm{CO}_{2}$ and $95 \%$ air at $37^{\circ} \mathrm{C}$. The cells were harvested following trypsinization $(0.025 \%$ trypsin and $0.02 \%$ EDTA) and washed twice with phosphate-buffered saline (PBS). When the cell density reached $\sim 80 \%$ confluence, the cells were subcultured, and SAC was then added to the cells following a 12-h incubation.

CCK-8 and EdU assay. The impact of SAC on prostate cell proliferation was measured by colorimetric cell proliferation assay using CCK-8, according to the manufacturer's instructions. Briefly, PC- 3 cells at $1 \times 10^{3}$ per well were cultured in 12 -well plates, divided into four groups, and treated with different concentrations of SAC $(0,5,10$ and $15 \mathrm{mM})$ for 5 days. Cell proliferation was assayed with CCK- 8 every $24 \mathrm{~h}$.

Cell proliferation was also tested by EdU incorporation assay using EdU assay kit (Ribobio, China), according to the manufacturer's instructions. Briefly, following a 5-day incubation, cells were exposed to $50 \mu \mathrm{mol} / 1 \mathrm{EdU}$ for an additional $2 \mathrm{~h}$ at $37^{\circ} \mathrm{C}$. After fixing with $4 \%$ formaldehyde and treating with $0.5 \%$ Triton X-100, the cells were reacted with $1 \mathrm{X}$ Apollo reaction cocktail for $30 \mathrm{~min}$. Subsequently, the DNA contents of the cells were stained with Hoechst $33342(5 \mu \mathrm{g} / \mathrm{ml})$ for $30 \mathrm{~min}$ and visualized under a fluorescence microscope.

Flow cytometric analysis. The cell cycle was determined by flow cytometry (FCM; Becton-Dickinson). Following incubation with SAC for 5 days, the cells were collected from the different groups, fixed with $70 \%$ ice ethanol for $24 \mathrm{~h}$, treated with $20 \mu \mathrm{g} / \mathrm{ml} \mathrm{RNase}$ A (Sigma-Aldrich, St. Louis, MO, USA), stained with $20 \mu \mathrm{g} / \mathrm{ml}$ propidium iodide (PI) (BD Pharmingen, San Diego, CA, USA), and analyzed using a flow cytometer (Beckman Coulter FC500) for cell cycle distribution.

Cell apoptosis was also tested by FCM using double staining with FITC-conjugated Annexin V and PI. Following incubation with SAC for 5 days, cells were harvested and incubated in a binding buffer containing Annexin V-FITC and PI at room temperature for $15 \mathrm{~min}$. Fluorescence analysis was performed using a flow cytometer.

Western blot analysis. Total protein was extracted according to the manufacturer's instructions (Sigma-Aldrich) and $50 \mu \mathrm{g}$ protein was loaded onto a 10\% SEMS-polyacrylamide gel and transferred to nitrocellulose filters. The filters were blocked with TBS-T buffer (10 mM Tris-HCl, $\mathrm{pH} 8.0,0.15 \mathrm{M} \mathrm{NaCl}$, $0.05 \%$ Tween-20) containing 5\% non-fat milk. The filters were then incubated with primary antibodies (Santa Cruz Biotechnology) overnight against Bcl-2, Bax and Caspase 8,
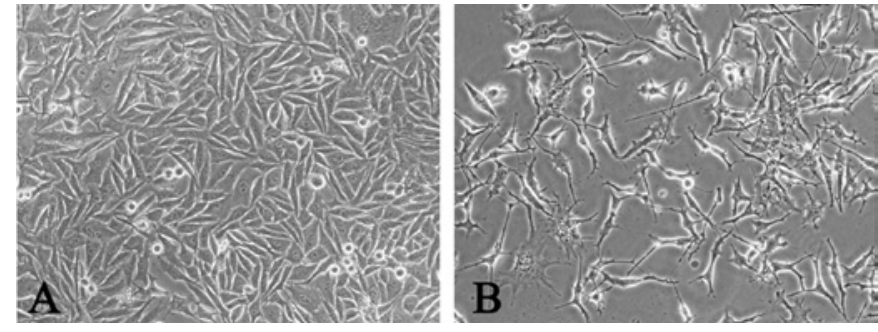

Figure 1. SAC induces morphological changes in PC-3 cells. (A) Normal PC-3 cells maintained a flat, spindle shape. (B) Following treatment with SAC, the cells typically exhibited a megagon, elongated shape. Microscopic images were captured at x100 magnification. SAC, S-allylcysteine.

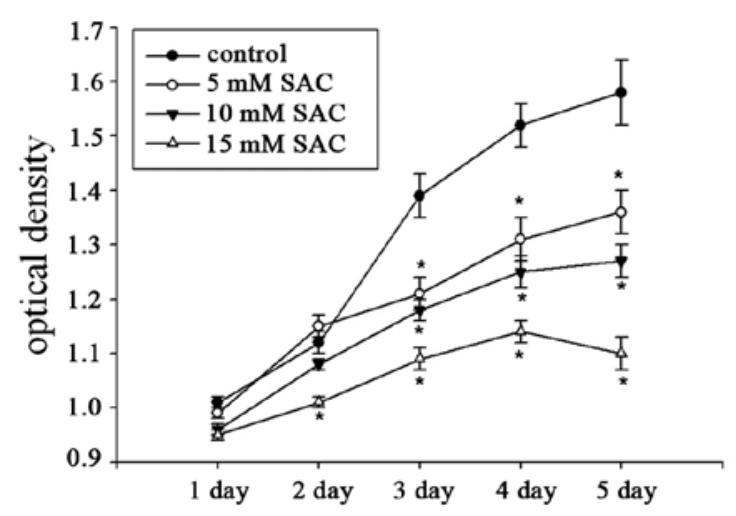

Figure 2. The different cell groups were treated with $0,5,10$ and $15 \mathrm{mM} \mathrm{SAC}$ for 5 days. Cell proliferation was tested by CCK- 8 assay. SAC inhibited cell proliferation in $\mathrm{PC}-3$ cells in a dose-dependent manner. Data are shown as the means \pm SEM. ${ }^{*} \mathrm{P}<0.05$ compared to the control group. SAC, S-allylcysteine.

followed by incubation with horseradish peroxidase-linked IgG. Signals were visualized using an enhanced chemiluminescence (ECL) Western blotting system (Amersham, Piscataway, NJ, USA). The expression of GAPDH was assessed as an internal loading control.

Statistical analysis. Experiments were repeated three times. All measurement data were expressed as the means \pm SEM. SPSS 12.0 statistical software (SPSS Inc., Chicago, IL, USA) was used for data analysis. The one-way analysis of variance (ANOVA) and the Student-Newman-Keuls (SNK) q-test were used to analyze the differences between groups. $\mathrm{P}<0.05$ was considered to be statistically significant.

\section{Results}

SAC inhibits cell proliferation in PC-3 cells. Firstly, PC-3 cells presented morphological changes following 5 days of incubation with SAC. The normal PC-3 cells maintained a flat, spindle shape (Fig. 1A), but the treated cells typically exhibited a megagon, elongated shape (Fig. 1B).

To test the effect of SAC on prostate cell proliferation, PC-3 cells were incubated with different concentrations of SAC, and the cell proliferative capacity was determined by CCK- 8 and EdU incorporation assays. As shown in Fig. 2, the growth of PC-3 cells in medium containing SAC was significantly inhibited compared to that in the control 
A
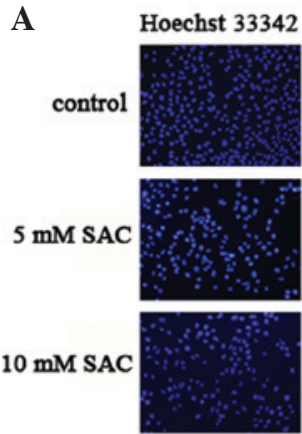

15 mM SAC
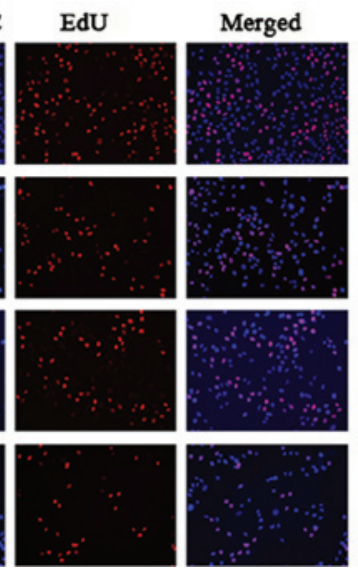

B

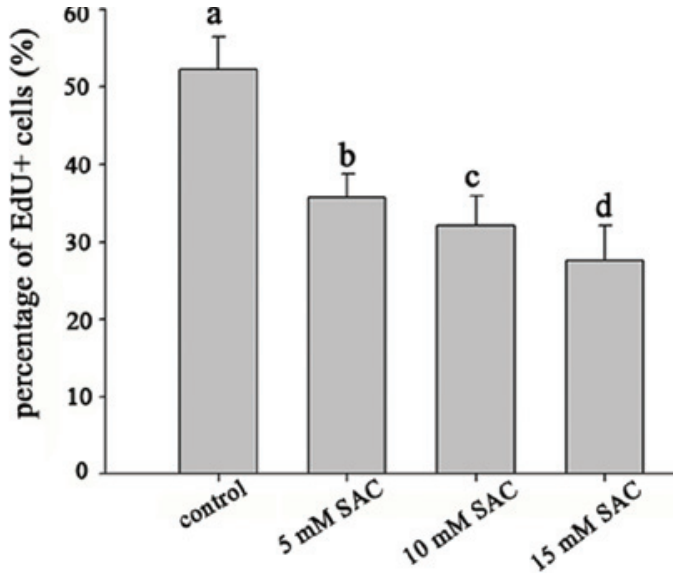

Figure 3. EdU incorporation assay was used to examine the inhibitory effects of SAC more sensitively and specifically. (A) EdU-labeled replicating cells were examined under a fluorescent microscope. Images were captured at x100 magnification. (B) The number of EdU ${ }^{+}$cells was significantly reduced in the SACtreated groups compared to the control. Data are from three independent experiments ( $\mathrm{n}=6$ per group). The values not sharing the same letter were significantly different $(\mathrm{P}<0.05)$. SAC, S-allylcysteine; EdU, 5-ethynyl-2'-deoxyuridine.

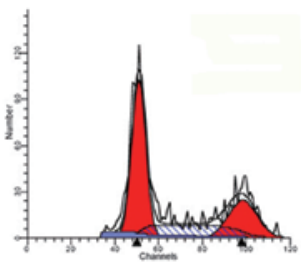

control

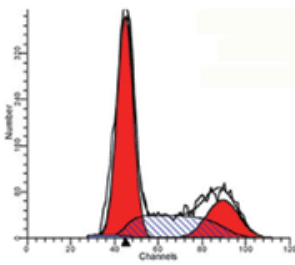

5 mM SAC

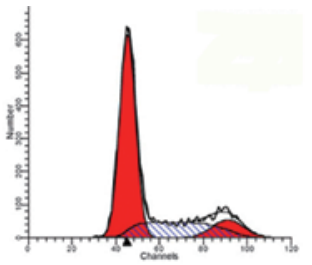

$10 \mathrm{mM} \mathrm{SAC}$

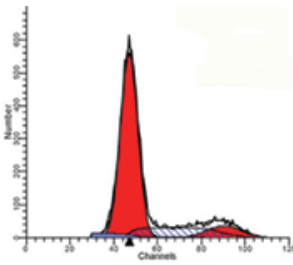

15 mM SAC

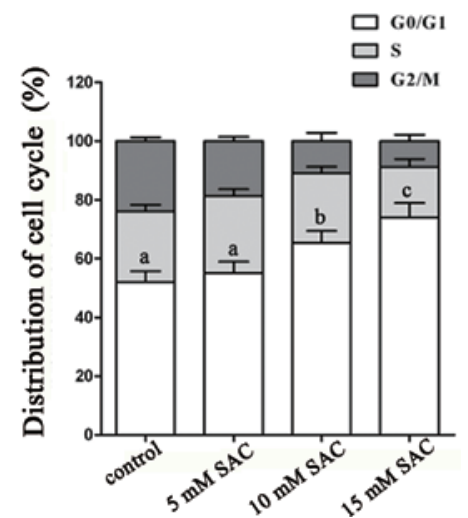

Figure 4. SAC induces PC-3 cell cycle arrest at the G0/G1 phases. PC-3 cells were treated with different concentrations of SAC for 5 days. Then, the cells were harvested and stained with propidium iodide, followed by FCM to assess the distribution of the cell cycle. Data are from three independent experiments ( $\mathrm{n}=6$ per group) and shown as the means \pm SEM. Values not sharing the same letter are significantly different $(\mathrm{P}<0.05)$. SAC, S-allylcysteine.
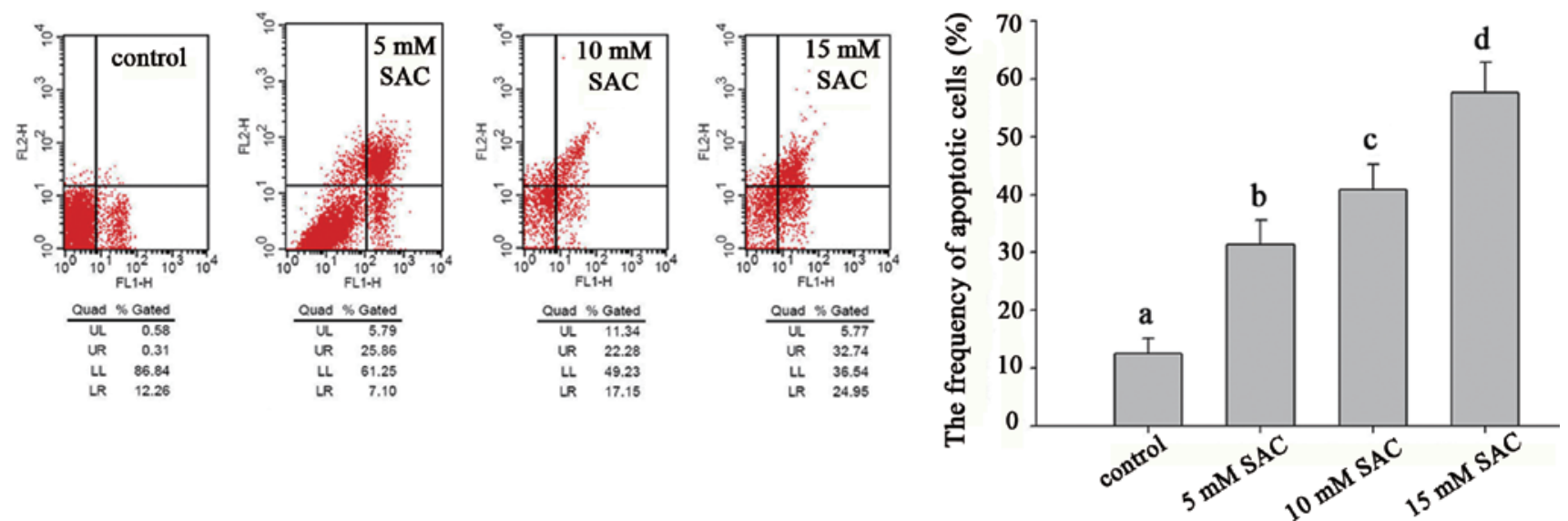

Figure 5. SAC induces cell apoptosis in PC-3 cells. The frequency of apoptotic cells (\%) treated with SAC (0-15 mM) for 5 days was analyzed by FCM. Data are from three independent experiments ( $\mathrm{n}=6$ per group) and shown as the means $\pm \mathrm{SEM}$. Values not sharing the same letter are significantly different $(\mathrm{P}<0.05)$ SAC, S-allylcysteine.

medium, and the inhibitory effect of SAC increased with increasing concentration. A significant statistical difference was observed between groups. Next, we used the EdU incorporation assay, a more sensitive and specific method, to examine the inhibitory effects of the different concentrations of SAC (Fig. 3A). Similarly, the number of EdU ${ }^{+}$cells was significantly reduced in SAC-treated groups compared to the control (Fig. 3B). 

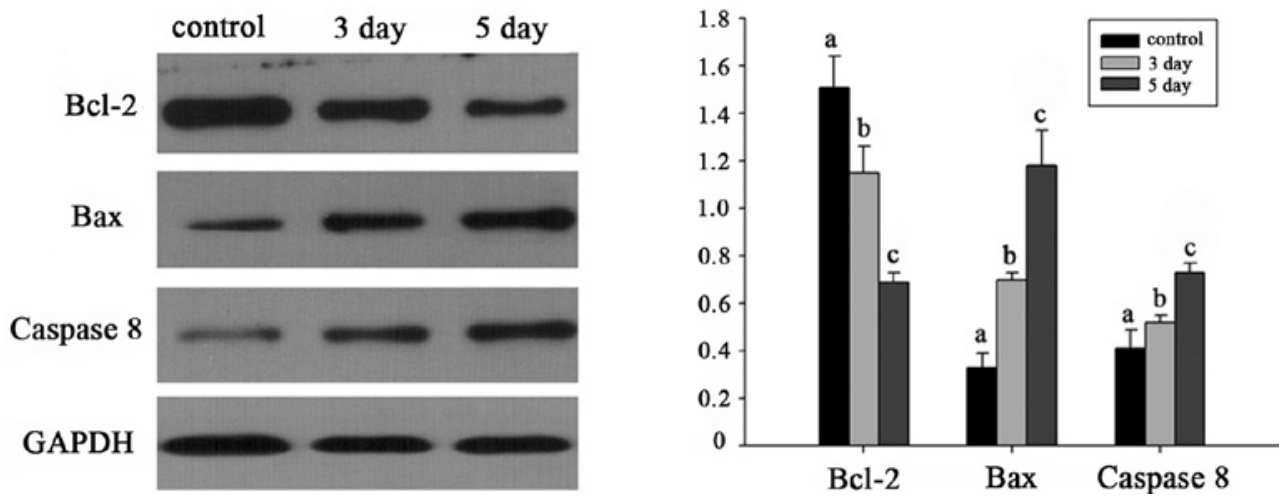

Figure 6. Expression of apoptosis-related proteins Bcl-2, Bax and caspase 8 was examined following incubation with SAC for 0, 3 and 5 days, respectively. Expression of Bcl-2 decreased, but the expression of Bax and caspase 8 increased in a time-dependent manner following treatment with SAC. Values not sharing the same letter are significantly different $(\mathrm{P}<0.05)$. SAC, S-allylcysteine.

SAC induces PC-3 cell cycling arrest at the G0/G1 phases. Cell cycling is associated with proliferation and its arrest leads to the inhibition of proliferation. To determine the impact of SAC on the cell cycle of prostate cancer cells, PC-3 cells were treated with different concentrations of SAC, and their cell cycle was characterized by FCM. The cells incubated with 10 and $15 \mathrm{mM} \mathrm{SAC}$ displayed a significantly higher frequency of cells at the G0/G1 phases, but there was no significant difference between the 5-mM SAC group and the control group (Fig. 4).

SAC induces cell apoptosis in PC-3 cells. The analysis of the different groups of cells revealed that the frequency of apoptotic cells was significantly higher in the SAC-treated groups than in the control group, and the pro-apoptotic effect of SAC increased with increasing concentration (Fig. 5).

Apoptosis-related proteins are regulated by SAC. To elucidate the possible mechanisms underlying the pro-apoptotic effect of SAC, the expression changes of apoptosis-related proteins Bcl-2, Bax and caspase 8 were examined by Western blot analysis. The expression of $\mathrm{Bcl}-2$ decreased, but the expression of Bax and caspase 8 increased in a time-dependent manner following treatment with SAC (Fig. 6).

\section{Discussion}

The aim of this study was to evaluate the anticancer effects of SAC on androgen-independent human prostate cancer cells and to elucidate the possible mechanisms. We used PC-3 cells as an in vitro model. First, we revealed that $\mathrm{SAC}$ suppressed the proliferation of PC-3 cells in a dose-dependent manner, and induced a higher frequency of arrested cells at the G0/ G1 phases. In conjunction with other studies into the antiproliferation function of SAC in prostate cancer $(17,18)$, our results provided additional support to the notion that SAC induces cell cycle arrest. Next, we found that SAC also induced cell apoptosis, and this effect was accompanied with decreased Bcl-2 expression and increased expression of Bax and caspase 8 .

Cell cycle checkpoints are critical in cancer pathogenesis, and may affect the effectiveness of cancer chemotherapy $(19,20)$. Numerous studies have suggested that cancer progression involves the loss of cell cycle checkpoint controls that regulate the passage through the cell cycle (21-24). Androgen exerts its actions through the androgen receptor (AR), which is crucial for the development and progression of prostate cancer (25). At the initial stage, androgen ablation inhibits cancer growth by inducing cell cycle arrest in prostate cancer cells (26). Under this selection pressure, certain cancer cells are driven to evolve mechanisms that restore AR activity and escape from cell cycle arrest under androgen-ablated conditions (27). This is a proposed mechanism of androgen-independent prostate cancer. Our data were consistent with a previous study that SAC inhibited the growth of androgen-independent prostate cancer cells (17). Next, we further examined the cell cycle phases. Our findings of the present study extended previous findings that SAC induced PC-3 cell arrest at the G0/G1 phases. We aimed to clarify the precise mechanism by which SAC regulates the cell cycle in androgen-independent prostate cancer cells.

A previous study demonstrated that SAC induces apoptosis in a CWR22R prostate cancer xenograft in vivo (18). Since the growth of prostate cancer cells is much more complex in vivo and many factors may affect cell growth, our present in vitro study is a supplement to the previous study. In accordance with our expectation, our data showed that SAC induced apoptosis of PC-3 cells in vitro. To analyze the potential mechanisms underlying the pro-apoptotic effect of SAC, we examined the apoptosis-associated proteins $\mathrm{Bcl}-2$, Bax and caspase 8 . We confirmed that SAC induced the decreased expression of Bcl-2 and the increased expression of Bax and caspase 8 . These results are similar to the findings of Velmurugan et al in experimental gastric cancer (28). The proteins of the Bcl-2 family play a primary role in regulating apoptosis by inducing a process called mitochondrial outer membrane permeabilization (MOMP) (29,30). If MOMP occurs, proteins present in the mitochondrial intermembrane space gain access to the cytosol and cause the activation of caspases, such as caspase 3 and 8 (31). The process of MOMP is antagonized by members of the anti-apoptotic Bcl-2 family, such as Bcl-2, Bcl-W, Bcl-xL and MCL-1, which inhibit the permeabilization function of Bax and Bak (32-34). Therefore, the ratio of Bcl-2/Bax is recognized as a good variable in predicting the propensity of a cell to undergo apoptosis. Our findings indicated that the $\mathrm{Bcl}-2 /$ Bax pathway may be a possible mechanism by which SAC induces the apoptosis of PC-3 cells. 
In conclusion, $\mathrm{SAC}$ reduced $\mathrm{PC}-3$ prostate cancer cell growth in vitro by inducing cell cycle arrest and apoptosis, and $\mathrm{Bcl}-2 / \mathrm{Bax}$ regulation may be involved in the tumor cell apoptosis induced by SAC. Our in vitro study indicated that SAC may be a potential phytochemical in the management of androgen-independent prostate cancer. Considering the relatively slow speed at which SAC takes effect, it may be more suitable for prevention, although in vivo study is required to confirm this.

\section{Acknowledgements}

This study was supported by a grant from the National Natural Sciences Foundation of China (no. 81001133) and the Graduates' Innovation Fund of Huazhong University of Science and Technology (no. HF-08-18-2011-540).

\section{References}

1. Jemal A, Siegel R, Xu J and Ward E: Cancer statistics, 2010. CA Cancer J Clin 60: 277-300, 2010.

2. Song F, He M, Li H, et al: A cancer incidence survey in Tianjin: the third largest city in China between 1981 and 2000. Cancer Causes Control 19: 443-450, 2008.

3. So A, Gleave M, Hurtado-Col A and Nelson C: Mechanisms of the development of androgen independence in prostate cancer. World J Urol 23: 1-9, 2005.

4. Mohla S, Stearns V, Sathyamoorthy N, Rosenfeld MG and Nelson P: The biology of hormone refractory breast and prostate cancer: an NCI workshop report. Cancer Biol Ther 8: 1975-1985, 2009.

5. Bhandari MS, Petrylak DP and Hussain M: Clinical trials in metastatic prostate cancer - has there been real progress in the past decade? Eur J Cancer 41: 941-953, 2005

6. Lim DY, Tyner AL, Park JB, Lee JY, Choi YH and Park JH: Inhibition of colon cancer cell proliferation by the dietary compound conjugated linoleic acid is mediated by the CDK inhibitor p21CIP1/WAF1. J Cell Physiol 205: 107-113, 2005.

7. Sliva D, Jedinak A, Kawasaki J, Harvey K and Slivova V: Phellinus linteus suppresses growth, angiogenesis and invasive behaviour of breast cancer cells through the inhibition of AKT signalling. Br J Cancer 98: 1348-1356, 2008.

8. Rivlin RS: Historical perspective on the use of garlic. J Nutr 131: S951-S954, 2001.

9. Park JH, Park YK and Park E: Antioxidative and antigenotoxic effects of garlic (Allium sativum L.) prepared by different processing methods. Plant Foods Hum Nutr 64: 244-249, 2009.

10. Ulbricht C, Chao W, Costa D, Rusie-Seamon E, Weissner W and Woods J: Clinical evidence of herb-drug interactions: a systematic review by the natural standard research collaboration. Curr Drug Metab 9: 1063-1120, 2008.

11. Asdaq SM and Inamdar MN: Potential of garlic and its active constituent, S-allylcysteine, as antihypertensive and cardioprotective in presence of captopril. Phytomedicine 17: 1016-1026, 2010.

12. Lee Y, Kim H, Lee J and Kim K: Anticancer activity of S-allylmercapto-L-cysteine on implanted tumor of human gastric cancer cell. Biol Pharm Bull 34: 677-681, 2011.

13. Tang FY, Chiang EP and Pai MH: Consumption of S-allylcysteine inhibits the growth of human non-small-cell lung carcinoma in a mouse xenograft model. J Agric Food Chem: Sept, 2010 (E-pub ahead of print).
14. Malki A, El-Saadani M and Sultan AS: Garlic constituent diallyl trisulfide induced apoptosis in MCF7 human breast cancer cells. Cancer Biol Ther 8: 2175-2185, 2009.

15. Cruz C, Correa-Rotter R, Sanchez-Gonzalez DJ, et al: Renoprotective and antihypertensive effects of S-allylcysteine in 5/6 nephrectomized rats. Am J Physiol Renal Physiol 293: F1691-F1698, 2007.

16. Katsuki T, Hirata K, Ishikawa H, Matsuura N, Sumi S and Itoh $\mathrm{H}$ : Aged garlic extract has chemopreventative effects on 1,2-dimethylhydrazine-induced colon tumors in rats. J Nutr 136: S847-S851, 2006.

17. Chu Q, Ling MT, Feng H, et al: A novel anticancer effect of garlic derivatives: inhibition of cancer cell invasion through restoration of E-cadherin expression. Carcinogenesis 27: 2180-2189, 2006.

18. Chu Q, Lee DT, Tsao SW, Wang X and Wong YC: S-allylcysteine, a water-soluble garlic derivative, suppresses the growth of a human androgen-independent prostate cancer xenograft, CWR22R, under in vivo conditions. BJU Int 99: 925-932, 2007.

19. Lapenna S and Giordano A: Cell cycle kinases as therapeutic targets for cancer. Nat Rev Drug Discov 8: 547-566, 2009.

20. Malumbres M and Barbacid M: Cell cycle, CDKs and cancer: a changing paradigm. Nat Rev Cancer 9: 153-166, 2009.

21. Caldon CE, Sutherland RL and Musgrove E: Cell cycle proteins in epithelial cell differentiation: implications for breast cancer. Cell Cycle 9: 1918-1928, 2010.

22. Zhang K, Wu J, Wu X, et al: p53R2 inhibits the proliferation of human cancer cells in association with cell-cycle arrest. Mol Cancer Ther 10: 269-278, 2011.

23. Brooke GN, Culley RL, Dart DA, et al: FUS/TLS is a novel mediator of androgen-dependent cell-cycle progression and prostate cancer growth. Cancer Res 71: 914-924, 2011.

24. Li B, Shi XB, Nori D, et al: Down-regulation of microRNA $106 \mathrm{~b}$ is involved in p21-mediated cell cycle arrest in response to radiation in prostate cancer cells. Prostate 71: 567-574, 2011.

25. Heinlein CA and Chang C: Androgen receptor in prostate cancer. Endocr Rev 25: 276-308, 2004.

26. Balk SP and Knudsen KE: AR, the cell cycle, and prostate cancer. Nucl Recept Signal 6: e001, 2008.

27. Debes JD and Tindall DJ: Mechanisms of androgen-refractory prostate cancer. N Engl J Med 351: 1488-1490, 2004.

28. Velmurugan B, Mani A and Nagini S: Combination of S-allylcysteine and lycopene induces apoptosis by modulating Bcl-2, Bax, Bim and caspases during experimental gastric carcinogenesis. Eur J Cancer Prev 14: 387-393, 2005.

29. Llambi F and Green DR: Apoptosis and oncogenesis: give and take in the BCL-2 family. Curr Opin Genet Dev 21: 12-20, 2011.

30. Tait SW and Green DR: Mitochondria and cell death: outer membrane permeabilization and beyond. Nat Rev Mol Cell Biol 11: 621-632, 2010.

31. Taylor RC, Cullen SP and Martin SJ: Apoptosis: controlled demolition at the cellular level. Nat Rev Mol Cell Biol 9: 231-241, 2008.

32. Reed JC: Proapoptotic multidomain Bcl-2/Bax-family proteins: mechanisms, physiological roles, and therapeutic opportunities. Cell Death Differ 13: 1378-1386, 2006.

33. Chipuk JE and Green DR: How do BCL-2 proteins induce mitochondrial outer membrane permeabilization? Trends Cell Biol 18: 157-164, 2008.

34. Knudson CM and Brown NM: Mitochondria potential, bax 'activation' and programmed cell death. Methods Mol Biol 414. 95-108, 2008. 